\title{
Open-loop position control in collaborative, modular Variable-Stiffness-Link (VSL) robots
}

\author{
Juan M. Gandarias ${ }^{1}$, Student Member, IEEE, Yongjing $\mathrm{Wang}^{2}$, Agostino Stilli ${ }^{2}$, Member, IEEE, \\ Alfonso J. García-Cerezo ${ }^{1}$, Member, IEEE, Jesús M. Gómez-de-Gabriel ${ }^{1}$, Member, IEEE, \\ Helge A. Wurdemann ${ }^{3}$, Member, IEEE
}

\begin{abstract}
Collaborative robots (cobots) open up new avenues in the fields of industrial robotics and physical Human-Robot Interaction (pHRI) as they are suitable to work in close approximation and in collaboration with humans. The integration and control of variable stiffness elements allow inherently safe interaction. Apart from notable work on Variable Stiffness Actuators, the concept of Variable-Stiffness-Link (VSL) manipulators promises safety improvements in cases of unintentional physical collisions. However, position control of these type of robotic manipulators is challenging for critical task-oriented motions (e.g., pick and place). Hence, the study of open-loop position control for VSL robots is crucial to achieve high levels of safety, accuracy and hardware cost-efficiency in pHRI applications. In this paper, we propose a hybrid, learning based kinematic modelling approach to improve the performance of traditional open-loop position controllers for a modular, collaborative VSL robot. We show that our approach improves the performance of traditional open-loop position controllers for robots with VSL and compensates for position errors, in particular, for lower stiffness values inside the links: Using our upgraded and modular robot, two experiments have been carried out to evaluate the behaviour of the robot during taskoriented motions. Results show that traditional model-based kinematics are not able to accurately control the position of the end-effector: the position error increases with higher loads and lower pressures inside the VSLs. On the other hand, we demonstrate that, using our approach, the VSL robot can outperform the position control compared to a robotic manipulator with 3D printed rigid links.
\end{abstract}

\section{INTRODUCTION}

Industrial robots have been particularly effective for fullyautomated processes in which typically high-payload machines are needed with a considerable robot body-mass in comparison with the average body mass of a human being [1]. For applications, in which hard automation is not possible and close collaboration with a human worker

This work is partially supported by the Spanish projects DPI2015-65186R, RTI2018-093421-B-I00, the University of Málaga, and the European Commission under grant agreement BES-2016-078237. The work is also supported by the Springboard Award of the Academy of Medical Sciences (grant number: SBF003-1109) and the Engineering and Physical Sciences Research Council (grant numbers: EP/N509577/1, EP/R037795/1, and $\mathrm{EP} / \mathrm{S} 014039 / 1)$

${ }^{1}$ J.M. Gandarias, A.J. García-Cerezo, and J.M. Gómez-de-Gabriel are with the Systems Engineering and Automation Department, University of Málaga, Spain. \{jmgandarias, ajgarcia, jesus.gomez @uma.es

${ }^{2}$ Y. Wang and A. Stilli are with the Department of Computer Science, University College London, UK. \{yongjing.wang.18, a.stilli\}@ucl.ac.uk

${ }^{3}$ H.A. Wurdemann is with the Department of Mechanical Engineering, University College London, UK. h. wurdemann@ucl.ac.uk

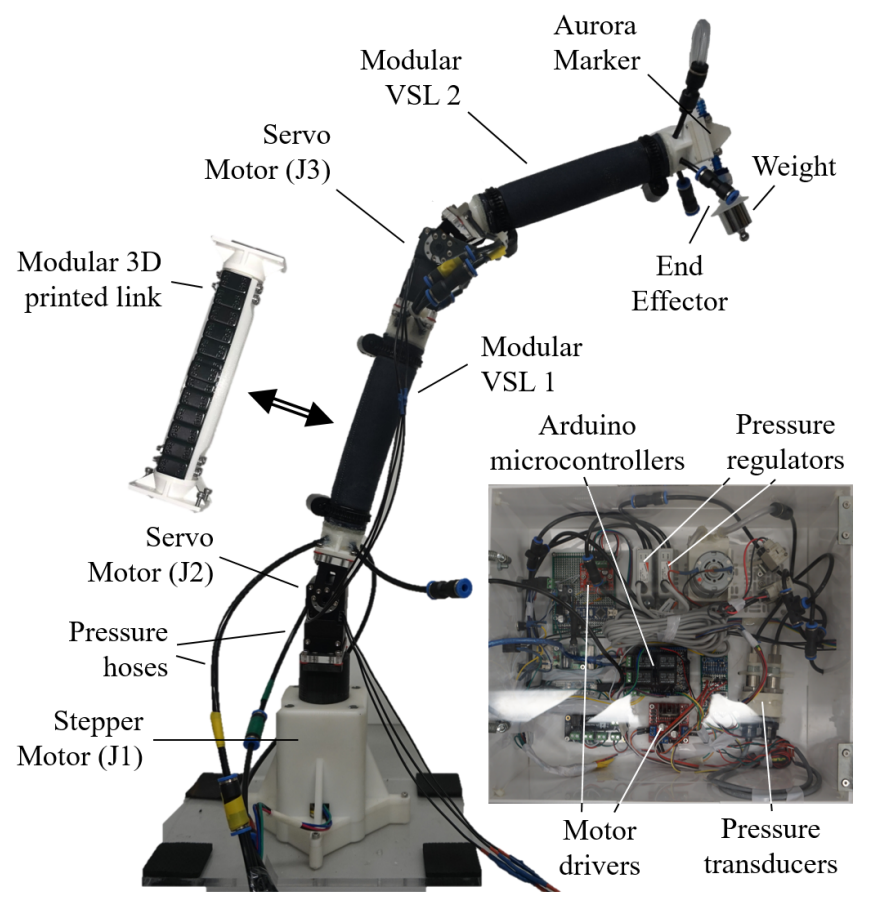

(a)

(b)

Fig. 1. (a) Enhanced prototype of our modular collaborative VSL robot: A stepper and servo motor allow 2 DoFs in the base. Two VSL are mounted in series connected by a second servo motor. An Aurora marker at the end-effector allow magnetic tracking of the manipulator's tip position. The VSL can be exchanged with 3D printed, rigid links of the same weight as the VSL. (b) Pressure regulators, transducers and motor controllers are interfaced using Arduino microcontrollers.

is necessary, industrial robots might potentially be harmful or life threatening to the human body [2], [1] - here, collaborative robots (cobots) offer advantages as integrated stiffness-controllable joints [3], sensing systems [4] and control strategies [5], which promise safe (physical) interaction. Cobots such as Universal Robots UR5/UR10 [6], the lightweight robots from KUKA [7], FerRobotics [8], or Franka [9] are made to work closely with humans without the need of safeguarding barriers. Electromechanical stiffnesscontrollable actuators, also called Variable Stiffness Actuators (VSA) [10], [11], adapt stiffness based on sensory information allowing safe Human Robot Interaction (HRI) according to ISO standards [12]. With the disruption introduced by soft material robots, VSAs have been advanced 
creating soft, stiffness-controllable actuators for applications in minimally invasive surgery [13].

Although notable work has been delivered to improve sensors and actuators performance for faster, safer and more accurate collision detection [14], limited efforts have been put into improving the intrinsic level of safety of the manipulators links. Passive solutions like soft coatings and skins have been developed to provide a softer contact surface (e.g., foam) in case of accidental collisions [15]. Recent research has considered the use of Variable Stiffness Links (VSL) to improve intrinsic safety in robotic manipulators. Instead of changing the stiffness locally using VSAs, the overall compliance of the robot can be controlled, resulting in an inherently safe configuration, in particular, when the mass of the robot is larger than the mass of the end-effector [16]. The concept of soft, stiffness-controllable links has been considered in previous works in combination with off-theshelf actuators to create a VSL robotic manipulator suitable for close collaboration with the human [17], [18]. A VSL consists of a combination of a silicone-based structure and reinforcing fabric material. Stiffness can be adjusted by controlling the pneumatic air pressure inside the link. A different concept of VSL that actively modulates stiffness via parallel, rotating beams has been recently presented [19]. Recent work in [20] considers a VSL made of multiple thin layers of rigid material and clamps. Here, stiffness is controlled by applying different clamping pressures.

Although safety is of paramount importance when closely collaborating with humans, position control is critical to perform tasks such as pick-and-place operations. Current studies have investigated the performance of a single VSL [21] and configuration combining the VSL with one Degree of Freedom (DoF) [22]. To the best of the authors knowledge, no work exists on exploring position control based on variable stiffness in VSL robotic manipulators.

Regarding position control of soft and continuum manipulators, some research studies propose the use of mechanical models that consider the deformation of the material and mechanical behaviour of the robot. Three main categories have been used: Piecewise Constant Curvature (PCC) [23], [24], classical Cosserat [25], [26], [27], and Finite Elements Models (FEM) [28]. Challenges remain, when applied to real physical robotics manipulators, with respect to real-time computation and accurate description of the kinematics, in particular, when external forces are exerted to a robot.

Other extended approach for soft robots control are based on kinematic models. The challenge of kinematic control of soft robots lies in complex models depending on a large number of parameters (e.g., actuation mechanism, materials, redundancy) [29], [30], [36], [32]. Three main approaches exist: model-based, model-free and hybrid kinematics [33]. Model-based kinematics consists of finding an analytical solution, while model-free solutions are based on experimentally collected data, typically using learning-based strategies. Hybrid modeling approaches combine both of these methodologies. The use of learning-based control techniques have been used in soft robotic systems [34] and for other applications, e.g., for a robotic manipulator navigating in an unstructured environment when in interacting with a human [35]. Open-loop position control only considers the internal state of the robot [36], whereas closed-loop requires additional external sensors such as cameras, that monitor the robot's state [23]. In real applications the integration of these kind of sensors is not always feasible.

In this paper, we apply learning methods to an open-loop position control for a modular VSL robot with 3 DoFs. In particular, the contributions of this work are:

- A hybrid, learning-based kinematic approach for openloop position control is embedded into a collaborative robot made of soft, stiffness-controllable links.

- The performance of open-loop position control, which varies according to the stiffness level of the links, is evaluated using traditional model-based kinematics and hybrid, learning based kinematic modelling.

- Our controller is integrated into an enhanced, modular Variable-Stiffness-Link (VSL) robot with sensing, control and actuation systems.

The advancements in relation to the VSL robot builds on our recent work presented in [18]: The sensing, control and actuation system has been enhanced allowing a complete study of the performance of open-loop position control when carrying out task-oriented motion. Two kinematic control strategies are embedded and compared: traditional modelbased kinematics and hybrid, learning based kinematic modelling. Two sets of experiments have been carried out to study the performance of the control strategies. The impact of our hybrid, learning based kinematic modelling approach is the improvement of the performance of traditional open-loop position controllers for robots with soft, stiffness-controllable links and the compensation of errors that can be achieved, in particular, for lower stiffness values of the links.

This paper is structured as follows: In Section II, the enhanced VSL robotic manipulator and interface is described. The traditional model-based kinematics and trajectory planning used for experimentation are presented in Section III. In Section IV, the learning-based kinematic model is explained. Finally, the experimental protocol and results as well as conclusions are described in sections V and VI respectively.

\section{ENHANCED MODULAR VSL ROBOTIC MANIPULATOR}

\section{A. The VSL robot - enhancing controllability and modularity}

The system presented in this work builds on the design presented by the authors in [18], where the very first integration of the VSL robot in a small scale anthropomorphic robotic platform was presented. The design presented in this work shares the same anthropomorphic configuration, however, the system presented here has been fitted with more advanced electro-mechanical components and sensors to reliably conduct a wide range of experiments.

To actuate joints $\mathrm{J} 2$ and $\mathrm{J} 3$ in the previous system $180^{\circ}$ HS-7954SH (Hitec RCD, Inc., Poway, CA, USA) servo motors had been used. To overcome the limitations of these motors in terms of torque, controllability and sensory 


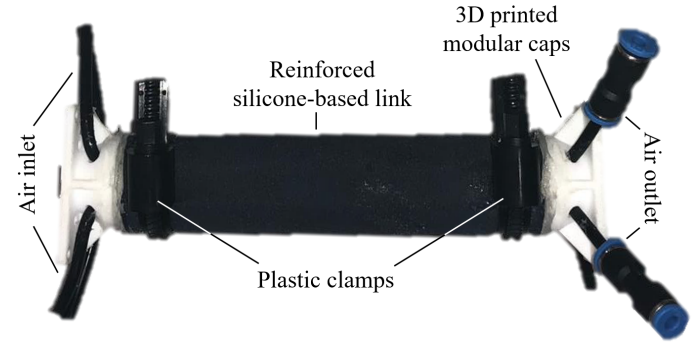

Fig. 2. The enhanced VSLs allow seamless modularity of VSL robots with up to three VSL. The fabric reinforced, silicone-based link (details in [17]) is sealed at each end using 3D printed caps. Fully integrated hoses supply additional VSL with pneumatic air pressure.

information, $360^{\circ}$ Dynamixels XM430-W350-T (ROBOTIS Inc.,Korea) servo motors were integrated here. These servo motors supply higher torque $(3.8 \mathrm{Nm})$ and angular position feedback. In addition, these Dynamixels servos allow the user to customise a number of control parameters inside the servo board, such as the PID gains, as well as to impose velocity and acceleration profiles. This results in having the possibility to validate our new system with higher payloads and in larger workspace. Further, the TTL Serial communication protocol allows the synchronous control of joints $\mathrm{J} 2$ and $\mathrm{J} 3$ with only one serial cable connection from joints $\mathrm{J} 3$ to $\mathrm{J} 2$, and joint $\mathrm{J} 2$ to the driver board, resulting in a more integrated robotic system.

Another critical limitation of the previous system had been represented by instability of the controller of the ITV00303BS pressure regulator (SMC Corporation, Tokyo, Japan) due to oscillating pressure values inside the VSLs. This oscillation affected the performance of the previous system adding noise on the pressure feedback, making the force estimation during interactions between the robot and the environment impossible. To overcome this limitation, an electromagnetic valve was placed in between the pressure regulator and the VSL pneumatic pressure line. The valve closes if the pressure is equal to the desired level, and allows air flow if the pressure drops. The pressure is read by the pressure sensor directly connected to VSL chambers. Thus, a stable pressure feedback is achieved. The robot here has now an active vacuum-based gripper mounted as the end effector. Given the advantages in weight and versatility, this type of gripper is preferred to electro-actuated mechanical ones.

Modularity is achieved by advanced VSL as shown in Figure 2: Multiple pressure hoses are embedded into the inner free cavity of the VSL. This allows to build manipulators with up to three VSL as well as exchanging any VSL with 3D printed rigid links as shown in Figure 1.

\section{B. Interfacing the VSL cobot}

A representative schematic of the control system in Figure 1(b) is shown in Figure 3. The stepper motor in the base is connected via a motor shield [A4988 Stepper Motor Driver] to an Arduino Nano microcontroller which again is linked to the main PC via serial communication. The two Dynamixel servo motors are driven by a U2D2 control board. A H-Bridge L298 board is used to covert Pulse Width Modulation (PWM) command signals from an Arduino Uno to $0-10 \mathrm{~V}$ analogue signals for two pressure regulators [Camozzi K8P-0-E522-0] and electromagnetic valves respectively. The analogue sensory feedback signals from the pressure transducers are first read by a 16 bits ADS1115 Analog/Digital converter, then read by the Arduino Uno through a I2C communication connection.

\section{MODEL-BASED KINEMATICS}

In this section, model-based kinematics based on rigid links and trajectory planning are described in order to compare its performance with a VSL robot.

\section{A. Kinematics}

Forward and inverse kinematics of a rigid anthropomorphic manipulator with 3 rotational DoFs has been solved and widely used in previous works [38], [37], [39]. The forward and inverse kinematics models used in this paper are defined by Equations (1-4).

$$
\begin{aligned}
{ }^{0} \mathbf{T}_{3}= & {\left[\begin{array}{cccc}
c_{1} c_{23} & -c_{1} s_{23} & s_{1} & c_{1}\left(L_{2} c_{2}+L_{3} c_{23}\right) \\
s_{1} c_{23} & -s_{1} s_{23} & -c_{1} & s_{1}\left(L_{2} c_{2}+L_{3} c_{23}\right) \\
s_{23} & c_{23} & 0 & L_{1}+L_{2} s_{2}+L_{3} s_{23} \\
0 & 0 & 0 & 1
\end{array}\right] } \\
\theta_{1} & =\arctan \left(\frac{Y}{X}\right) \\
\theta_{2} & =\arctan \left(\frac{r_{2}}{r_{1}}\right)+\lambda \arccos \left(\frac{r_{3}^{2}+L_{2}^{2}-L_{3}^{2}}{2 r_{3} L_{2}}\right) \\
\theta_{3} & =\pi+\lambda \arccos \left(\frac{L_{2}^{2}+L_{3}^{2}-r_{3}^{2}}{2 L_{2} L_{3}}\right)
\end{aligned}
$$

where $c_{i}=\cos \left(\theta_{i}\right), s_{i}=\sin \left(\theta_{i}\right), c_{i j}=\cos \left(\theta_{i}+\theta_{j}\right)$ and $s_{i j}=$ $\sin \left(\theta_{i}+\theta_{j}\right), \lambda$ defines the configuration of the robot as elbow up $(\lambda=1)$ or elbow down $(\lambda=-1)$, the joint space is defined as $\mathbf{q}=\left[\theta_{1}, \theta_{2}, \theta_{3}\right]$ and $r_{1}, r_{2}$ and $r_{3}$ are the distances defined in Equations 5-7.

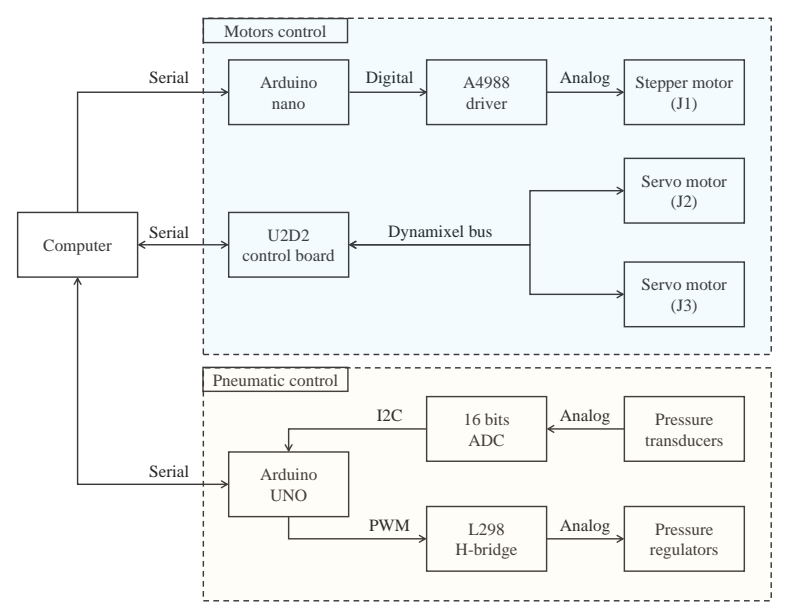

Fig. 3. Representative schematic of the control system in Figure 1(b). 


$$
\begin{aligned}
& r_{1}=\sqrt{X^{2}+Y^{2}} \\
& r_{2}=Z-L_{1} \\
& r_{3}=\sqrt{r_{1}^{2}+r_{2}^{2}}
\end{aligned}
$$

\section{B. Jacobian}

Like the kinematics, it is well known that the Jacobian of the manipulator can be calculated by direct differentiation from the forward kinematics. In this work, the Jacobian of the manipulator is defined in Equation (8).

$$
\mathbf{J}(\mathbf{q})=\left[\begin{array}{ccc}
-s_{1}\left(L_{2} c_{2}+L_{3} c_{23}\right) & -c_{1}\left(L_{2} s_{2}+L_{3} s_{23}\right) & -L_{3} c_{1} s_{23} \\
c_{1}\left(L_{2} c_{2}+L_{3} c_{23}\right) & s_{1}\left(L_{2} s_{2}+L_{3} s_{23}\right) & -L_{3} s_{1} c_{23} \\
0 & L_{2} c_{2}+L_{3} c_{23} & L_{3} c_{23}
\end{array}\right]
$$

\section{Trajectory planning}

In this work, a point-to-point trajectory planning is used. The trajectories described below are used to analyse the performance of the robot.

1) Linear trajectory: The linear trajectory algorithm (see Algorithm 1) describes a linear movement that starts at initial point $\mathbf{x}_{0}$ and ends at goal point $\mathbf{x}_{g}$, with a constant velocity $\dot{\mathbf{x}}_{g}$. The first step of the algorithm consists of moving the endeffector to the initial position with the initial joint velocities $\dot{\mathbf{q}}_{0}$. Then, for each point $k$, from a total of $N$ points, the algorithm calculates the coordinates of the next point $\mathbf{x}_{k+1}(i)$, where $i$ represents the three Cartesian coordinates of the point and the joint velocities $\dot{\mathbf{q}}_{k}$ using the inverse Jacobian $\mathbf{J}^{-1}\left(\mathbf{q}_{k}\right)$. After that, the motors move to the next position.

2) Circular trajectory: The circular trajectory algorithm is defined in Algorithm 2, and describes a circular movement from an initial point $\mathbf{x}_{0}$ to a goal point $\mathbf{x}_{g}$, knowing the center of the circular trajectory $\mathbf{x}_{c}$ and the desired velocity $V$. The trajectory is defined in the $X Z$ plane, so the $y$ coordinate of the next point $\mathbf{x}_{k+1}(2)$ is always 0 .

\section{HYBRID, LEARNING-BASED KINEMATICS}

The methodology described in this section combines traditional model-based kinematics, presented in the previous section, with a learning model based on deep neural networks (NN), to improve the performance of both forward and inverse kinematics.

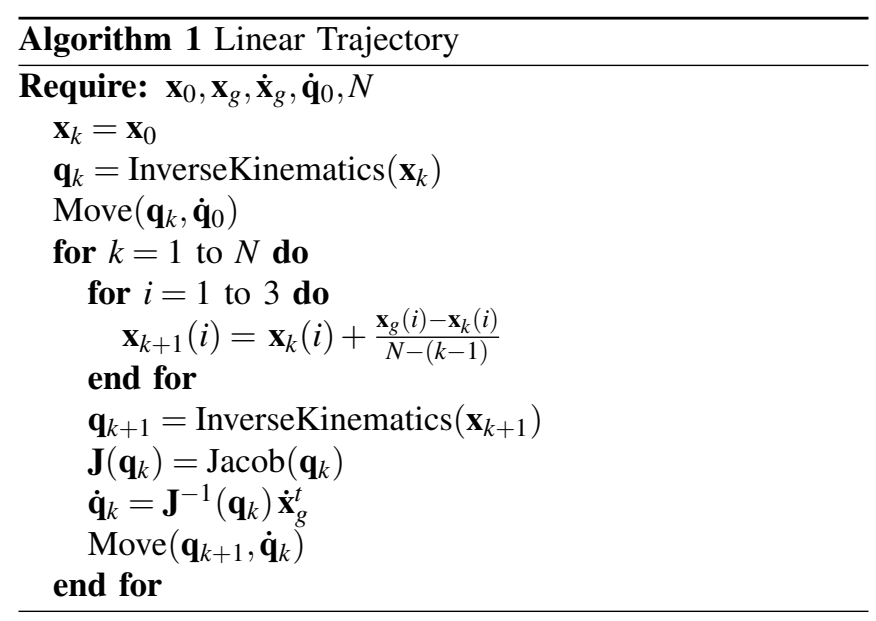

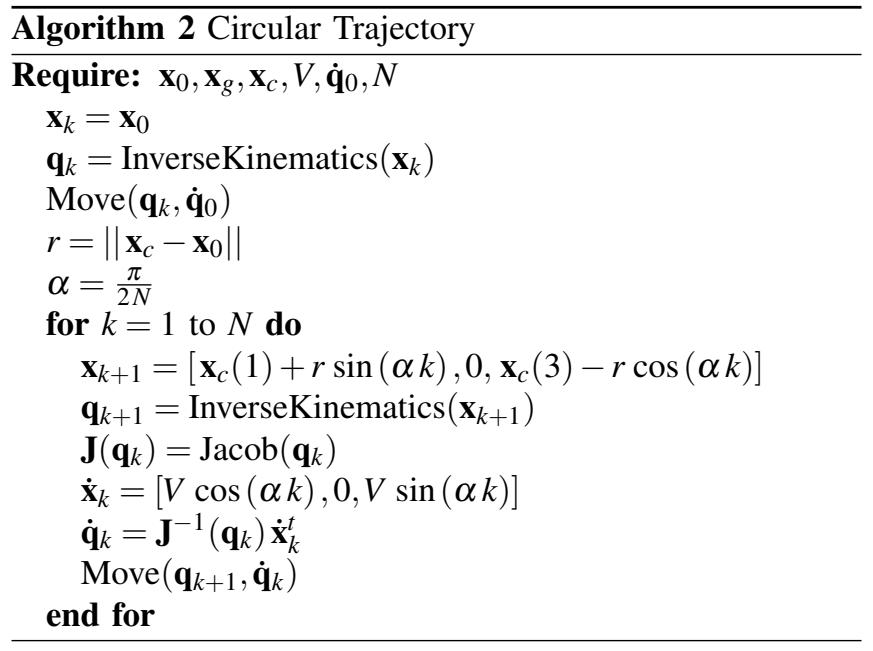

\section{A. Dataset collection}

To train the learning system, we have collected a dataset which contains data from multiple points of the workspace with different pressures and loads. The Aurora 3D Tracking system (NDI Intl. Ontario, Canada) was used to measure the actual position of the end-effector as ground-truth data for the machine learning methods.

The dataset is represented by the matrix D: each row has information about the ground truth position in Cartesian space $\left(\mathbf{X}_{i}\right)$ measured with the tracking system the internal pressure of the links $\left(\mathbf{p}_{i}\right)$, the current of the motors $\left(\mathbf{i}_{i}\right)$ and the position of the servos $\left(\mathbf{q}_{i}\right)$.

$$
\mathbf{D}=\left[\begin{array}{cccc}
\mathbf{x}_{1} & \mathbf{p}_{1} & \mathbf{i}_{1} & \mathbf{q}_{1} \\
\vdots & \vdots & \vdots & \vdots \\
\mathbf{x}_{n} & \mathbf{p}_{n} & \mathbf{i}_{n} & \mathbf{q}_{n}
\end{array}\right]
$$

The dataset collection process has been carried out recording data from encoders and electrical current sensors of the servos, pressure sensors of the links and the tracking system. In this respect, the workspace has been reduced due to the limited range of the tracking system. A total of $n=17568$ points have been collected. Each point is reached by randomly varying the pressure, servo position or load in each step.

\section{B. Forward model}

The hybrid, learning-based forward model (see Figure 4) outputs the predicted position in Cartesian space $\left(\tilde{\mathbf{X}}_{k}\right)$ using the position resulted from the model-based forward kinematics $\left(\mathbf{X}_{k}\right)$, the internal pressure of the links $\left(\mathbf{p}_{k}\right)$, and the current of the motors $\left(\mathbf{i}_{k}\right)$ as inputs.

Hence, the model can be represented as a non-linear function $(\mathcal{L})$ that estimates the position of the end-effector according to Equation (10).

$$
\tilde{\mathbf{X}}_{\mathbf{k}}=\mathcal{L}\left(\mathbf{X}_{k}, \mathbf{p}_{k}, \mathbf{i}_{k}\right)
$$

The model uses a 7 layer $\mathrm{NN}$ as an estimator, which has 7 inputs and 3 outputs. All the layers are fully-connected, and the activation functions of every layer are ReLUs. The 


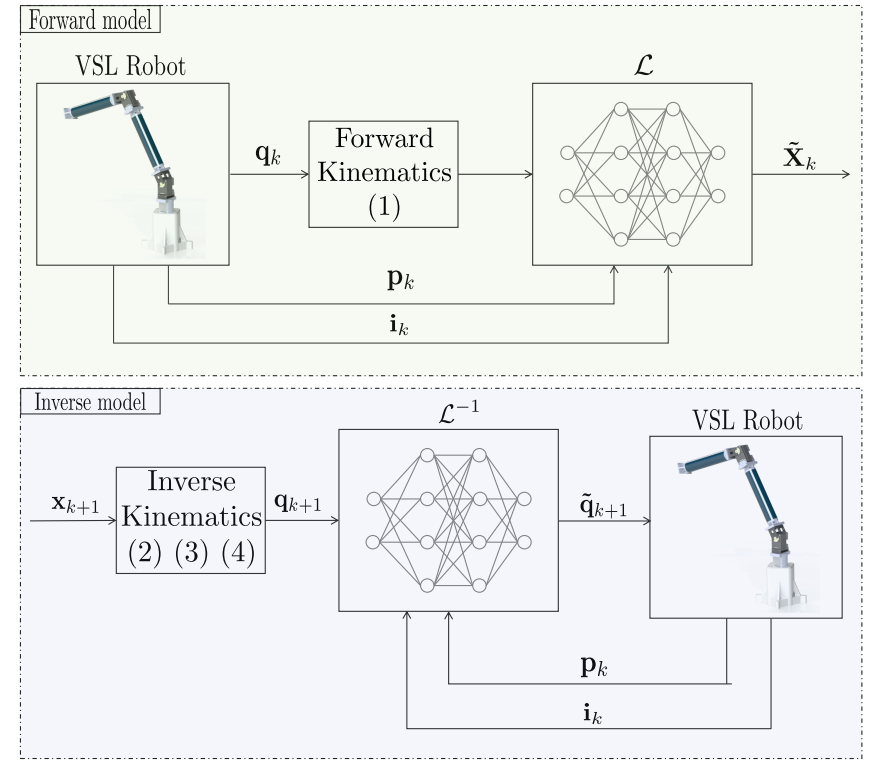

Fig. 4. Schematic of the hybrid model: Forward learning-based kinematics (top) and inverse learning-based kinematics (bottom).

number of neurons from layer 1 to layer 7 is $64,256,1024$, 1024, 256 and 64 respectively. The integration of this model has been done using the deep learning toolbox of Matlab R2019a. To train the model, $\mathbf{p}_{k}, \mathbf{i}_{k}$, and $\mathbf{q}_{k}$ from matrix D are used as inputs, whereas $\mathbf{x}_{k}$ is used as the desired output. Training, validation and test sets are chosen randomly, where the training set contains $70 \%$ of the data, and validation and test sets contains $15 \%$ each.

\section{Inverse model}

The hybrid, learning-based inverse model presented in Figure 4 is similar to the forward model. In this case, an NN estimates the next desired servo positions $\left(\tilde{\mathbf{q}}_{k+1}\right)$ from the next position obtained by the model-based Inverse Kinematics $\left(\mathbf{q}_{k+1}\right), \mathbf{p}_{k}$, and $\mathbf{i}_{k}$. In other words, considering the current pressure of the links and motors current, the system outputs a corrected servo position that compensates the error that might be produced by the weight of the arm and the soft links.

The learning-based inverse kinematics can be described as a non-linear function $\left(\mathcal{L}^{-1}\right)$ that predicts compensated servo positions according to Equation (11).

$$
\tilde{\mathbf{q}}_{k+1}=\mathcal{L}^{-1}\left(\mathbf{q}_{k+1}, \mathbf{p}_{k}, \mathbf{i}_{k}\right)
$$

The NN architecture is the same as in the learning-based forward kinematics, but the training hyperparameters have changed to achieve a good performance.

\section{EXPERIMENTAL PROTOCOL, RESULTS AND DISCUSSION}

In this section, the results of the experiments are presented and discussed. Two experiments have been carried out to analyze the performance of the robot when following different trajectories. In order to better represent the paths, only two motors (J2 and J3) are used for experimentation, keeping J1 to 0 . Therefore, the robot moves on the plane $\mathrm{XZ}$, as we can presume that there is no position error in axis $\mathrm{Y}$. The position error is due to the weight and internal pressure and we can also assume that they have effects on axes $\mathrm{X}$ and $\mathrm{Z}$ only.

\section{A. Experiment 1: Model-based kinematics}

In the first experiment, the performance of the system, using traditional model-based kinematics, is studied using different configurations of loads and pressures and compared with a fully-rigid manipulator with same dimensions and parameters when following different trajectories.

Three trajectories have been programmed: a linear trajectory between $\mathbf{x}_{0}=[300,0,50]$ and $\mathbf{x}_{g}=[300,0,350]$; a curved trajectory which describes two semicircles, the first one with $\mathbf{x}_{0}=[300,0,50], \mathbf{x}_{g}=[400,0,150]$ and $\mathbf{x}_{c}=[300,0,150]$, and the second one with $\mathbf{x}_{0}=[400,0,150], \mathbf{x}_{g}=[300,0,50]$ and $\mathbf{x}_{c}=[400,0,50]$; and a square trajectory that is composed by four linear trajectories between points $\mathbf{x}_{0}=[350,0,50]$, $\mathbf{x}_{1}=[250,0,50], \mathbf{x}_{2}=[250,0,150]$ and $\mathbf{x}_{3}=[350,0,150]$.

\section{B. Experiment 2: Hybrid model}

In this experiment, the model-based kinematics and the hybrid, learning-based models are compared. The curved trajectory of Experiment 1, with low and high pressures and loads, is used to study the behaviour of the robot.

\section{Results and Discussion}

The results of Experiment 1 are presented in Figure 5. Columns, from left to right, show the behaviour of the manipulator when the end-effector is loaded with increasing weights starting from no load to $100 \mathrm{~g}$, in $25 \mathrm{~g}$ intervals. The blue and red plots represent the mean trajectories and standard deviations of the robot's end-effector based on the servo motor position reading and rigid links combined with the model-based forward kinematics. The remaining plots show the end-effector the trajectories when the VSLs are pressurised by 0.5 bar (green), 1 bar (purple), 1.5 bar (yellow) and 2 bar (orange). The blue plot represents the positions of the end-effector according to the measured joint angles (servo encoders) and the model-based forward kinematics, which is similar to the desired path. The trajectory of a fully rigid manipulator (red) should be consistent with the servo paths (blue). However, there are some position error on the rigid manipulator due to small deformations of the 3D printed links, the arm weight and loads. As expected, the position errors using the pressurised VSLs are larger. As expected, errors also increase with higher payloads and lower VSL pressures. Besides, the error also depends on the arm pose, as can be seen in Figure 5(c): The further the end-effector is from the base of the robot, the higher the error is.

The results of Experiment 2 are shown in Figure 6. The end-effector load and VSL pressure are $25 \mathrm{~g}$ and $1.5 \mathrm{bar}$ (Figure 6 left) and $75 \mathrm{~g}$ and 0.5 bar (Figure 6 right). The green plot shows the model-based path. The actual trajectory (mean and standard deviation) is shown in blue. The purple plot shows the trajectory estimated by the hybrid model. 

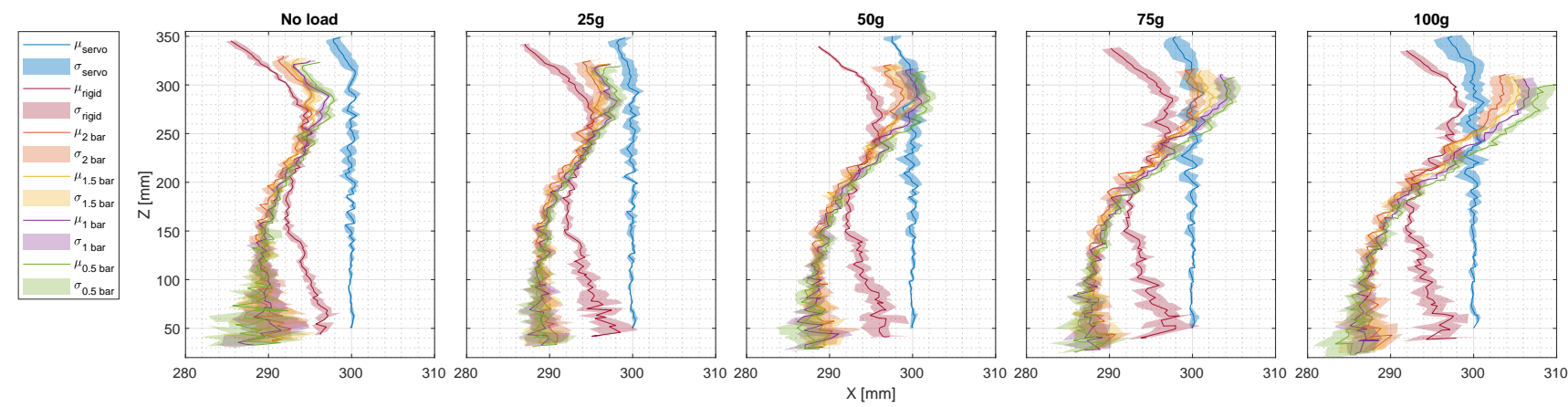

(a)
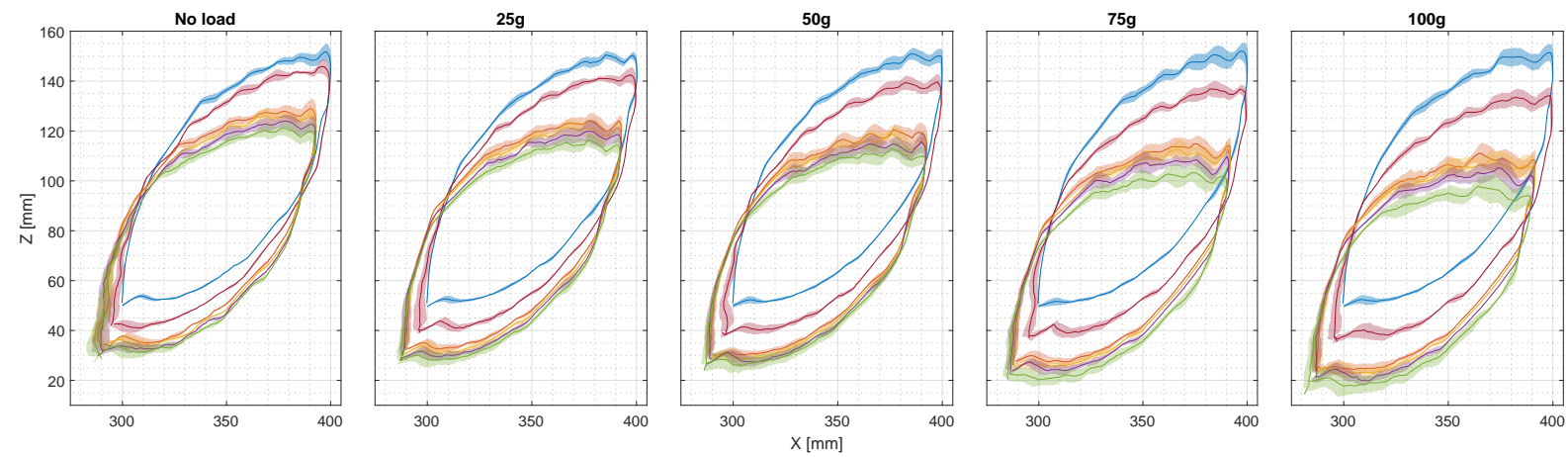

(b)
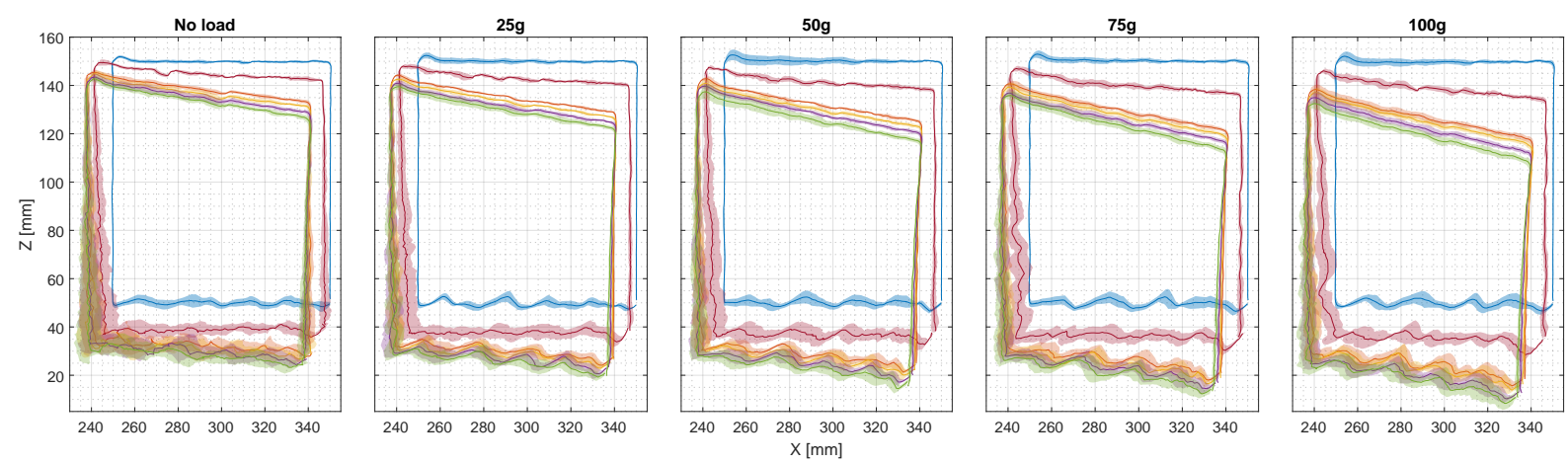

(c)

Fig. 5. Results of experiment 1: (a) Linear Trajectory, (b) Curve Trajectory, and (c) Square Trajectory. For each trajectory, the actual positions of the end-effector using rigid links and soft links with different pressures and loads has been recorded using the tracking system. For each case, the trajectory is commanded 5 times, and the mean $(\mu)$ and standard deviation $(\sigma)$ are represented. Servo positions indicate the path according to the model-based forward kinematics and the measurements from the encoders.

Compared to the results of Experiment 1, the performance has been improved by the addition of the learning-based model both in forward and inverse cases. Figure 6 further demonstrates that the hybrid forward model can predict the real position of the end-effector more precisely than the model-based kinematic model.

Figure 7(a) shows how the desired path can be followed by the VSL arm using the hybrid inverse model for two load/pressure combinations. The actual paths using both the model-based inverse kinematics and the hybrid inverse model are compared with the desired path in a single execution. Here, the green and red plots show the mean trajectories of the model-based approach and hybrid model respectively. The hybrid inverse model provides a compensation trajectory (dashed line), so the actual positions are closer to the desired path even with soft links. The position errors are shown in Figure 7(b) including the mean, range and a box between the $25^{\text {th }}$ and $75^{\text {th }}$ percentile. The position error has been calculated as the distance between the points of the measured paths and the desired path. Figure 7 shows improvements in open-loop position control when using the hybrid inverse model. It can be observed that the path followed using the inverse hybrid model achieve lower position errors than using the model-based inverse kinematics. In fact, Figure 7(b) concludes that the position errors of the VSL robotic manipulator controlled by the hybrid model are lower than those obtained with an arm that uses rigid links. 


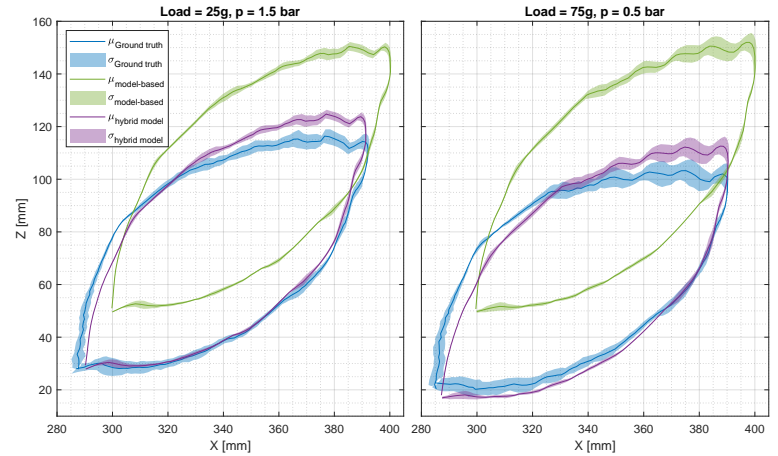

Fig. 6. Expected path according to the model-based forward kinematics and the hybrid forward model when following the curve trajectory of Experiment 1, and a comparison with the real path.

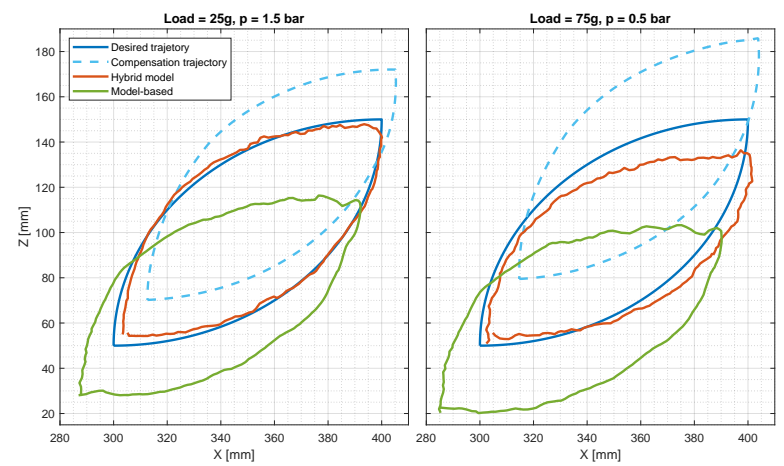

(a)

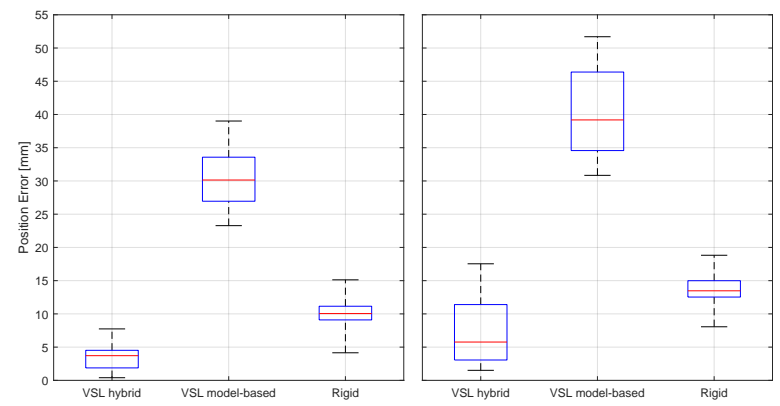

(b)

Fig. 7. (a) Comparison of the paths followed using the model-based inverse kinematics and the hybrid, learning-based inverse model. (b) Representation and comparison of the position errors achieved by the VSL manipulator with model-based and hybrid approaches, and the rigid arm.

\section{CONCLUSIONS}

In this paper, the performance of open-loop position control in VSL robots has been studied. An enhanced, modular version of the 3-DoF VSL robot published in [18] has been presented, and the new control, actuation and sensing systems have been described. Two modeling approaches have been explained and implemented in the real system: traditional kinematic models, and hybrid, learning-based models. The model-based forward kinematics has been solved considering the Denavit-Hartenberg notation, whereas the inverse kinematics has been solved by geometric methods, both using only the measurements from the encoders and disregarding the deformation of the links. Besides, no external sensors are required, compared to when using closed-loop control techniques, and, hence, the complexity and overall cost of the system is not affected. The implementation of the hybrid model has consisted of estimating the real position in the case of the forward model, and predicting a compensated next position in the case of the inverse model, given the output from the model-based kinematics and using it as an input of a $\mathrm{NN}$ along with the measures of the internal pressure of the links and the current of the motors. Two experiments have been carried out to analyze the behaviour of the system according to both approaches. In Experiment 1 , the performance of the robot when controlled based on traditional inverse kinematic model has been studied, showing that the position error increases when the robot bends due to high loads and low pressures. On the other hand, in Experiment 2, it has been proved that the use of $\mathrm{NN}$ that considers the expected position given by traditional kinematics and the data from other proprioceptive sensors helps to improve both open-loop control with the inverse model and the estimation of the real position with the forward model.

In future works the integration of other sensors like IMUs can be considered to get more helpful information for the hybrid models not only for position control but for the physical interaction with humans or the environment. The use of time-series of data and spatio-temporal, learning-based techniques may also be contemplated. Also, the comparison of this work to closed-loop control techniques, and the integration of more complex mechanical models will be considered for real applications with a scaled-up VSL robot. Moreover, the path planning and trajectory following might be improved by using more advanced models considering the dynamics effects or close-loop strategies.

\section{REFERENCES}

[1] A. De Santis, B. Siciliano, A. De Luca, A. Bicchi, "An atlas of physical human-robot interaction", Mechanism and Machine Theory, vol. 43(3), pp. 253-270, 2008.

[2] H.A. Yanco, J. L. Drury, "A Taxonomy for Human-Robot Interaction", Engineering, p. 9, 2002.

[3] E. Magrini, F. Flacco, A, De Luca, "Control of generalized contact motion and force in physical human-robot interaction", IEEE International Conference on Robotics and Automation, pp. 2298-2304, 2015.

[4] K. Kronander, A. Billard, "Learning compliant manipulation through kinesthetic and tactile human-robot interaction", IEEE transactions on haptics, vol. 7(3), 367-380, 2013.

[5] J.M. Gandarias, F. Pastor, A.J. Muñoz-Ramírez, A.J. García-Cerezo, J.M. Gómez-de-Gabriel, "Underactuated Gripper with Forearm Roll Estimation for Human Limbs Manipulation in Rescue Robotics", IEEE/RSJ International Conference on Intelligent Robots and Systems, 2019.

[6] "Universal Robots website (retrieved on 14.08.2019)." [Online]. Available: http://www.universal-robots.com.

[7] "KUKA Robots website (retrieved on 14.08.2019)." [Online]. Available: http://www.kuka-robotics.com.

[8] "FerRobotics Compliant Robot Technology website (retrieved on 14.08.2019)." [Online]. Available: http://www.ferrobotics.com.

[9] "FRANKA EMIKA website (retrieved on 14.08.2019)." [Online]. Available: https://www.franka.de.

[10] G. Tonietti, R. Schiavi, A. Bicchi, "Design and Control of a Variable Stiffness Actuator for Safe and Fast Physical Human Robot Interaction", IEEE International Conference on Robotics and Automation, pp. 526-531, 2005. 
[11] R. Ham, T. Sugar, B. Vanderborght, K. Hollander, D. Lefeber, "Compliant actuator designs", IEEE Robotics and Automation Magazine, vol. 16(3), 2009.

[12] "ISO/TS 15066:2016. Robots and robotic devices - Collaborative robots (retrieved on 23.08.2019)." [Online]. Available: https://www.iso.org/standard/62996.html

[13] L. Paterno, G. Tortora, A. Menciassi, "Hybrid Soft-Rigid Actuators for Minimally Invasive Surgery”, Soft Robotics, vol.5(6), pp. 783-799, 2018.

[14] C. Yang, G. Ganesh, S. Haddadin, S. Parusel, A. Albu-Schaeffer, E. Burdet, "Human-like adaptation of force and impedance in stable and unstable interactions", IEEE Transactions on Robotics, vol. 27(5), pp. 918-930, 2011.

[15] "Comau website (retrieved on 14.08.2019)." [Online]. Available: https://www.comau.com.

[16] Y. She, D. Meng, J. Cui, H.J. Su, "On the impact force of human-robot interaction: Joint compliance vs. link compliance", IEEE International Conference on Robotics and Automation, pp. 6718-6723, 2017.

[17] A. Stilli, H.A. Wurdemann, K. Althoefer, "A novel concept for safe, stiffness-controllable robot links", Soft Robotics, vol.4(1), pp. 16-22, 2017.

[18] A. Stilli, L. Grattarola, H. Feldmann, H.A. Wurdemann, K. Althoefer, "Variable Stiffness Link (VSL): Toward inherently safe robotic manipulators", IEEE International Conference on Robotics and Automation, pp. 4971-4976, 2017.

[19] T. Morrison, C. Li, X. Pei, H.J. Su, Hai-Jun, "A Novel Rotating Beam Link for Variable Stiffness Robotic Arms", IEEE International Conference on Robotics and Automation, pp. 9387-9393, 2019.

[20] Y. Zhou, L.M. Headings, M.J. Dapino, ”Discrete Layer Jamming for Safe Co-Robots", IEEE International Conference on Robotics and Automation, pp. 6124-6129, 2019.

[21] S. Song, X. Zeng, Y. She, J. Wang, H.J. Su, "Modeling and control of inherently safe robots with variable stiffness links", Robotics and Autonomous Systems, vol.120, pp. 103247, 2019.

[22] M.T. Gillespie,C.M. Best, M.D. Killpack, Marc D., "Simultaneous position and stiffness control for an inflatable soft robot", IEEE International Conference on Robotics and Automation, pp. 1095-1101, 2016.

[23] T.G. huruthel, E. Falotico, F. Renda, C. Laschi, C., "Model-based reinforcement learning for closed-loop dynamic control of soft robotic manipulators", IEEE Transactions on Robotics, vol.35(1), pp. 124-134, 2018.

[24] R.K. Katzschmann, C. Della Santina, Y. Toshimitsu, A. Bicchi, D. Rus, "Dynamic motion control of multi-segment soft robots using piecewise constant curvature matched with an augmented rigid body model", IEEE International Conference on Soft Robotics, pp.454-461, 2019.

[25] D. Trivedi, C.D. Rahn, "Model-based shape estimation for soft robotic manipulators: The planar case", Journal of Mechanisms and Robotics, vol.6(2), 021005, 2014.

[26] F. Renda, F. Boyer, J. Dias, L. Seneviratne, ”Discrete cosserat approach for multisection soft manipulator dynamics", IEEE Transactions on Robotics, vol.34(6), pp. 1518-1533, 2018.

[27] H.B. Gilbert, I.S. Godage, "Validation of an Extensible Rod Model for Soft continuum Manipulators", IEEE International Conference on Soft Robotics (RoboSoft), pp. 711-716, 2019.

[28] R.K. Katzschmann, M. Thieffry, O. Goury, A. Kruszewski, T.M Guerra, C. Duriez, D. Rus, "Dynamically Closed-Loop Controlled Soft Robotic Arm using a Reduced Order Finite Element Model with State Observer", IEEE International Conference on Soft Robotics (RoboSoft), pp. 717-724, 2019.

[29] A. Ataka, A. Stilli, J. Konstantinova, H.A. Wurdemann, K. Althoefer, "Kinematic Control and Obstacle Avoidance for Soft Inflatable Manipulator", Annual Conference Towards Autonomous Robotic Systems, Lecture Notes in Computer Science, vol. 11649, Springer, Cham, pp. 52-64, 2019.

[30] A. Stilli, E. Kolokotronis, J. Fraś, A. Ataka, K. Althoefer, H.A. Wurdemann, "Static Kinematics for an Antagonistically Actuated Robot Based on a Beam-Mechanics-Based Model", IEEE/RSJ International Conference on Intelligent Robots and Systems, pp. 6959-6964, 2018.

[31] T.G. Thuruthel, C. Laschi, M. Cianchetti, E. Falotico, A. Pratesi, M. Manti, "Learning Closed Loop Kinematic Controllers for Continuum Manipulators in Unstructured Environments", Soft Robotics, vol.4(3), pp. 285-296, 2017.

[32] T.G. Thuruthel, E. Falotico, M. Manti, C. Laschi, "Stable Open
Loop Control of Soft Robotic Manipulators", IEEE Robotics and Automation Letters, vol.3(2), pp. 1292-1298, 2018.

[33] T.G. Thuruthel, Y. Ansari, E. Falotico, C. Laschi, "Control Strategies for Soft Robotic Manipulators: A Survey", Soft Robotics, vol.5(2), pp 149-163, 2018

[34] J.D. Ho, K.H. Lee, W.L. Tang, K.M. Hui, K. Althoefer, J. Lam, K.W. Kwok, "Localized online learning-based control of a soft redundant manipulator under variable loading", Advanced Robotics, vol. 32(21), pp. 1168-1183, 2018

[35] S. Calinon, I. Sardellitti, D.G. Caldwell, "Learning-based control strategy for safe human-robot interaction exploiting task and robot redundancies", IEEE/RSJ International Conference on Intelligent Robots and Systems, pp. 249-254, 2010

[36] T.G. Thuruthel, E. Falotico, F. Renda, C. Laschi, "Learning dynamic models for open loop predictive control of soft robotic manipulators", Bioinspiration \& biomimetics, vol.12(6), 066003, 2017.

[37] C.S.G. Lee, M. Ziegler, "Geometric approach in solving inverse kinematics of PUMA robots", IEEE Transactions on Aerospace and Electronic Systems, vol.6, pp. 695-706, 1984

[38] R.P. Paul "Kinematic control equations for simple manipulators", IEEE Transactions on Systems, Man and Cybernetics, vol.11(6), pp. 449455, 1981.

[39] C.R. Rocha, C.P. Tonetto, A. Dias, "A comparison between the Denavit-Hartenberg and the screw-based methods used in kinematic modeling of robot manipulators", Robotics and Computer-Integrated Manufacturing, vol.27(4) pp. 723-728, 2011.

[40] R.S. Hartenberg, and J. Denavit, Jacques, "A kinematic notation for lower pair mechanisms based on matrices", Journal of applied mechanics, vol.77, pp. 215-221, 1955. 